\title{
Myocardial remodeling in rats with metabolic syndrome: role of Rho-kinase mediated insulin resistance
}

\author{
Chuan-Bao Li#, Xiao-Xing Li#, Yu-Guo Chen ${ }^{1,4, \bowtie}, \mathrm{Hai}-Q i n g \mathrm{Gao}^{2}$, Cheng-Mei Bao³, Xiang-Qun \\ $\mathrm{Liu}^{2}$, Pei-Li Bu${ }^{4}$, Juan Zhang ${ }^{4}$, Yun Zhang ${ }^{4}$ and Xiao-Ping Ji4, ${ }^{4}$ \\ 'Department of Emergency, Shandong University Qilu Hospital, Jinan, Shandong China; 2Department of Geriatrics, Shandong University Qilu \\ Hospital, Jinan, Shandong, China; ${ }^{3}$ Department of Internal Medicine, Jinan Second People's Hospital, Jinan, Shandong, China; ${ }^{4 T h e ~ K e y ~ L a b o r a-~}$ \\ tory of Cardiovascular Remodeling and Function Research, Chinese Ministry of Education and Chinese Ministry of Public Health, Shandong \\ University Qilu Hospital, Jinan, Shandong, China
}

Insulin resistance (IR) plays a critical role in metabolic syndrome (MS). Previous studies have demonstrated that activated ROCK is increased in MS patients. However, the effect of Rho-kinase (ROCK) on IR has not been definitely determined. Thus, the aims of the present study were to determine whether ROCK activation induces IR or affects myocardial structure and function, as well as the possible mechanisms underlying this process. Wistar rats fed high fat, high glucose and high salt diet sewed as model of MS and we used transmission electron microscopy, echocardiogram technology, and terminal deoxynucleotidyl transferase-mediated DNA nick-end labeling staining to identify any myocardial damage. The protein levels of MYPT-1 (characteristic of ROCK activation), IRS1 and AKT were analyzed by immunohistochemistry and Western blotting. In hearts from MS rats, we found increased protein levels of phospho-MYPT-1 and phosphoIRS-1 (Ser307) and decreased phospho-AKT compared to levels in normal rats. In conclusion, the results suggest that ROCK-mediated IR is involved in the development of myocardial impairments in MS rats and that this effect is mediated probably via the IRS-1/PI3-kinase/AKT pathway.

Key words: Insulin resistance; Phosphorylation; Myocardium; Metabolism; Rat

Received: 18 August, 2011; revised: 07 May, 2012; accepted: 03 June, 2012; available on-line: 11 June, 2012

\section{INTRODUCTION}

Insulin resistance (IR) plays an important role in the pathophysiology of metabolic syndrome (MS) (Bonora, 2006; Fan \& Peng, 2007). The insulin signaling pathway is activated once insulin binds to its membrane-bound tyrosine kinase receptor, leading to subsequent phosphorylation of intracellular insulin receptor substrates (IRSs). The phosphorylated IRSs are then used as docking sites for downstream proteins to further transduce the insulin signal through the PI3-kinase/AKT pathway (Ferre, 2007). PI3-kinase/AKT activation is essential and collectively controls programmed cell death, cellular metabolism and organ protection (Amaravadi \& Thompson, 2005; Asano et al., 2007). The IR mechanism involves activation of several serine/threonine kinases, reduction in tyrosine phosphorylation of IRS-1/2, and impairment of the IRS/PI3-kinase pathway of insulin signaling (Boden, 2006; Sesti, 2006).
Rho-associated kinase (ROCK) is a serine/threonine protein kinase that is activated by RhoA, a member of the Rho family of small G-proteins (Noma et al., 2006). Abnormal RhoA/ROCK pathway activation has been observed in major cardiovascular disorders, such as atherosclerosis, restenosis, hypertension and cardiac hypertrophy (Chitaley et al., 2001; Fonseca, 2003; Loirand et al., 2006). ROCK was activated in rats with spontaneous and angiotensin II-induced hypertension, and treatment with ROCK inhibitors effectively reduced blood pressure (Mukai et al., 2001; Uehata et al., 1997). Furthermore, increased RhoA/ROCK pathway activation has been demonstrated in both type 2 diabetic $\mathrm{db} / \mathrm{db}$ mice and streptozotocin-induced diabetic rats (Bivalacqua et al., 2004; Xie et al., 2006). Previous studies have already shown that ROCK activity is increased in the serum from MS patients (Liu et al., 2007) and in arteries from obese Zucker rats (a model of MS) (Naik et al., 2006). Additionally, ROCK-mediated IR either positively or negatively regulates insulin signaling, depending on cell type and stimulus (Shi \& Wei, 2007). It is currently unknown, however, whether increased ROCK activity is also present in the hearts of MS patients.

Therefore, we induced MS in rats with special diets that cause insulin resistance. The present study show that ROCK was activated in hearts from MS rats, and that activation of ROCK-mediated IR induced changes of cardiac structure and function during chronic MS possibly through the IRS/PI3-kinase/AKT pathway in vivo.

\section{MATERIALS AND METHODS}

Animals. All experiments were carried out in accordance with the recommendations of the Universities Federation for Animal Welfare Handbook on the Care and Management of Laboratory Animals. Twenty-two male Wistar rats from the animal center of Shandong University ( 6 weeks of age; mean body weight $135 \pm 10 \mathrm{~g}$ ) were used. The rats were caged under standard light con-

e-mail: xiaopingji64@hotmail.com

\#These authors contributed equally to this work.

Abbreviations: BW, body weight; $\mathrm{CHO}$, cholesterol; FBG, fasting blood glucose; HDL-C, high-density lipoprotein cholesterol; IHC, immunohistochemical; IR, insulin resistance; IVSW, intraventricular septal wall; LDL-C, low-density lipoprotein cholesterol; LV, left ventricle; LVPW, left ventricular posterior wall thickness; MYPT-1, myosin phosphatase targeting subunit 1; MS, metabolic syndrome; ROCK, Rho-kinase; SBP, systolic blood pressure; TEM, transmission electron microscopy; TG, triglycerides; TUNEL, terminal deoxynucleotidyl transferase-mediated dUTP nick end labeling. 
ditions (alternating $12 \mathrm{~h}$ light/dark cycles), temperature $\left(22 \pm 0.5^{\circ} \mathrm{C}\right)$ and humidity $(60 \pm 10 \%)$ during all experiments. The rats were then randomly divided into two groups. Twelve were fed with free access to a high-fat, high-glucose and high-salt diet with sucrose solution to induce MS (MS group). Concomitantly, ten age-matched Wistar rats were fed normal diet as controls (NC group). After 28 weeks, rats with MS $(n=10)$ were identified using criteria analogous to ATP III as $\geq 3$ of the following: hypertriglyceridemia, low HDL-C, high fasting glucose, excessive waist circumference, and hypertension.

Basic characteristics and plasma data analysis. Body weight (BW) was measured every two weeks in the morning, together with systolic blood pressure (SBP) by the tail-cuff method using a Rat Tail Manometer provided by the Japanese and Chinese Friendly Hospital (RBT-1, Beijing, China). Samples of venous blood were collected every two weeks to detect triglycerides (TG), cholesterol (CHO), HDL-C, LDL-C, fasting blood glucose (FBG) and insulin (FINS) levels after 12 hours of fasting. The insulin resistance index (IRI) was calculated as follows: IRI $=\left(F_{B}{ }^{*}\right.$ FINS) $/ 22.5$. FBG, CHO, HDL-C, LDL-C, TG and FINS were measured in a clinical laboratory of Qilu Hospital, which is affiliated with Shandong University (Jinan, China).

Echocardiographic evaluation. At the begining of the study and before sacrifice, each rat was anaesthetized with chloral hydrate $(0.3 \mathrm{ml} / 100 \mathrm{~g})$ through intraperitoneal injection, and placed in the left lateral decubitus position. Echocardiography was performed with Hewlett-Packard equipment, Sonos 7500 model, and an electronic $10-\mathrm{MHz}$ transducer. M-mode recordings were obtained of the left ventricle $(\mathrm{LV})$ at the level of mitral valve in the parasternal view using two-dimensional echocardiographic guidance in both the short and long axis views. Pulsed wave Doppler was used to examine mitral diastolic inflow from the apical four chamber view. Integrated backscatter images in the parasternal long-axis view also were recorded that quantifies the intensity of the backscattered echoes returning from myocardial cells within a user-defined region of interest. For each measurement, data from three consecutive cardiac cycles were averaged. All measurements were made from digital images captured at the time of the study by use of inherent analysis software.

Evaluation of collagen deposition. Paraffin-embedded tissue sections $(5 \mu \mathrm{m})$ of the median part of the left ventricle were hydrated and stained with Masson Accustain Trichrome stain (Sigma, St. Louis, MO). The proportion of blue staining, indicative of interstitial collagen, was measured in 10 fields randomly selected on each of the three non-consecutive serial sections from each heart, and the values were averaged. Additionally, five- $\mu$ m-thick paraffin sections were stained with the collagen-specific Sirius Red for measurement of the interstitial fibrosis.

TUNEL stainning. The terminal deoxynucleotidyl transferase-mediated dUTP nick end labeling (TUNEL) technique was used to evaluate apoptotic activity. Each section was deparaffinized and rehydrated. Proteinase $\mathrm{K}$ (20 $\mathrm{mg} / \mathrm{L})$ was applied to the section for $1 \mathrm{~h}$ with the intention of producing optimal proteolysis. The endogenous peroxidase was inhibited with $3 \%$ hydrogen peroxide for $10 \mathrm{~min}$. A commercial apoptosis detection kit (Roche, Germany) was used. The TdT reaction was carried out for $1 \mathrm{~h}$ at $37^{\circ} \mathrm{C}$, and then $\mathrm{DAB}$ chromogen was applied. The sections were analyzed with a computerassisted color image analysis system (Image-ProPlus 5.1, Media Cybernetics, Silver Spring, MD, USA).
Immunohistochemical (IHC) staining. Myocardial tissues were collected from both groups and immediately fixed in 4\% paraformaldehyde solution for 24 hours. The tissues were then dehydrated and embedded in paraffin using standard histological procedures. Paraffin sections were dewaxed and rehydrated before microwave antigen retrieval. To retrieve the antigen, slides were soaked in $1 \%$ citric acid buffer, $\mathrm{pH} 6.0$ and kept at $92-98^{\circ} \mathrm{C}$ for 10-15 min. The slides were cooled at room temperature for $30 \mathrm{~min}$, rinsed with PBS, and blocked in $5 \%$ bovine serum albumin for $20 \mathrm{~min}$. Slides were then incubated overnight at $4^{\circ} \mathrm{C}$ with $1: 100$ rabbit polyclonal anti-rat phospho-AKT antibody (Cell Signaling Technology), and with 1:200 rabbit polyclonal anti-rat total-AKT antibody (Cell Signaling Technology), followed with 1:200 biotinylated anti-rabbit IgG secondary antibody (Santa Cruz Biotechnology). DAB substrate kits were used to develop the immunohistochemical reaction. PBS was substituted for the primary antibody as a negative control.

Transmission electron microscopy (TEM). A $0.5 \times 1 \times 5 \mathrm{~mm}^{3}$ cross section from cardiac muscle tissues was fixed with $2 \%$ glutaraldehyde for electron microscopic examination. The tissues were examined for any cardiocyte changes using an $\mathrm{H}-7000 \mathrm{FA}$ transmission electron microscope (Hitachi Co. Ltd.) at the Academy of Medical Science (Jinan, Shandong, China).

Western blot. ROCK activity was assessed by examining the phosphorylation state of myosin phosphatase targeting subunit 1 (MYPT-1), a well-established ROCKspecific substrate. Myocardial MYPT-1, IRS-1 and AKT protein levels were determined by western blot analysis. Equal amounts of protein $(50 \mu \mathrm{g})$ were separated on $10 \%$ SDS-polyacrylamide gels in running buffer (25 $\mathrm{mM}$ Tris, $192 \mathrm{mM}$ glycine, $0.1 \%$ (w/v) SDS, $\mathrm{pH} 8.3$ ) at $8 \mathrm{~V} / \mathrm{cm}$ and electroblotted onto nitrocellulose membranes. The membranes were blocked at room temperature with 5\% nonfat milk in Tris-buffered saline $(25 \mathrm{mM}$ Tris, $137 \mathrm{mM} \mathrm{NaCl}, 2.7 \mathrm{mM} \mathrm{KCl}$ ) containing $0.05 \%$ Tween-20 (TBS-T) and incubated overnight at $4^{\circ} \mathrm{C}$ with the following primary antibodies: rabbit polyclonal antirat phospho-MYPT-1 (Thr853) antibody (Santa Cruz Biotechnology; 1:1500 dilution; molecular weight of $\mathrm{p}$ MYPT-1: $130 \mathrm{kDa}$ ), rabbit polyclonal anti-rat phosphoMYPT-1 (Thr696) antibody (Cell Signaling Technology; 1:1500 dilution; molecular weight of p-MYPT-1: 140 $\mathrm{kDa}$ ), rabbit polyclonal anti-rat MYPT-1 antibody (Cell Signaling Technology; 1:1000 dilution; molecular weight of MYPT-1: $140 \mathrm{kDa}$, rabbit polyclonal anti-rat phospho-IRS-1 (Ser307) antibody (Cell Signaling Technology; 1:1000 dilution; molecular weight of p-IRS-1: $180 \mathrm{kDa}$ ), rabbit polyclonal anti-rat IRS-1 antibody (Cell Signaling Technology; 1:1000 dilution; molecular weight of IRS1: $180 \mathrm{kDa}$, rabbit polyclonal anti-rat phospho-AKT (Ser473) antibody (Cell Signaling Technology; 1:1000 dilution; molecular weight of p-AKT: $60 \mathrm{kDa}$ ), rabbit polyclonal anti-rat AKT antibody (Cell Signaling Technology; 1:1000 dilution; molecular weight of AKT: $60 \mathrm{kDa}$ ), or $\beta$-actin (Santa Cruz Biotechnology; 1:1000 dilution; molecular weight of $\beta$-actin: $43 \mathrm{kDa}$ ). The membranes were then washed three times in TBS-T and incubated with horseradish peroxidase-conjugated goat anti-rabbit secondary antibodies (Santa Cruz Biotechnology; 1:10,000 dilution) at room temperature. Immunoreactive bands were visualized using an enhanced chemiluminescence substrate and quantified with an image analyzer (AlphaImager 2200).

Statistical analysis. Data was expressed as mean \pm S.D. Experimental means were subjected to either 


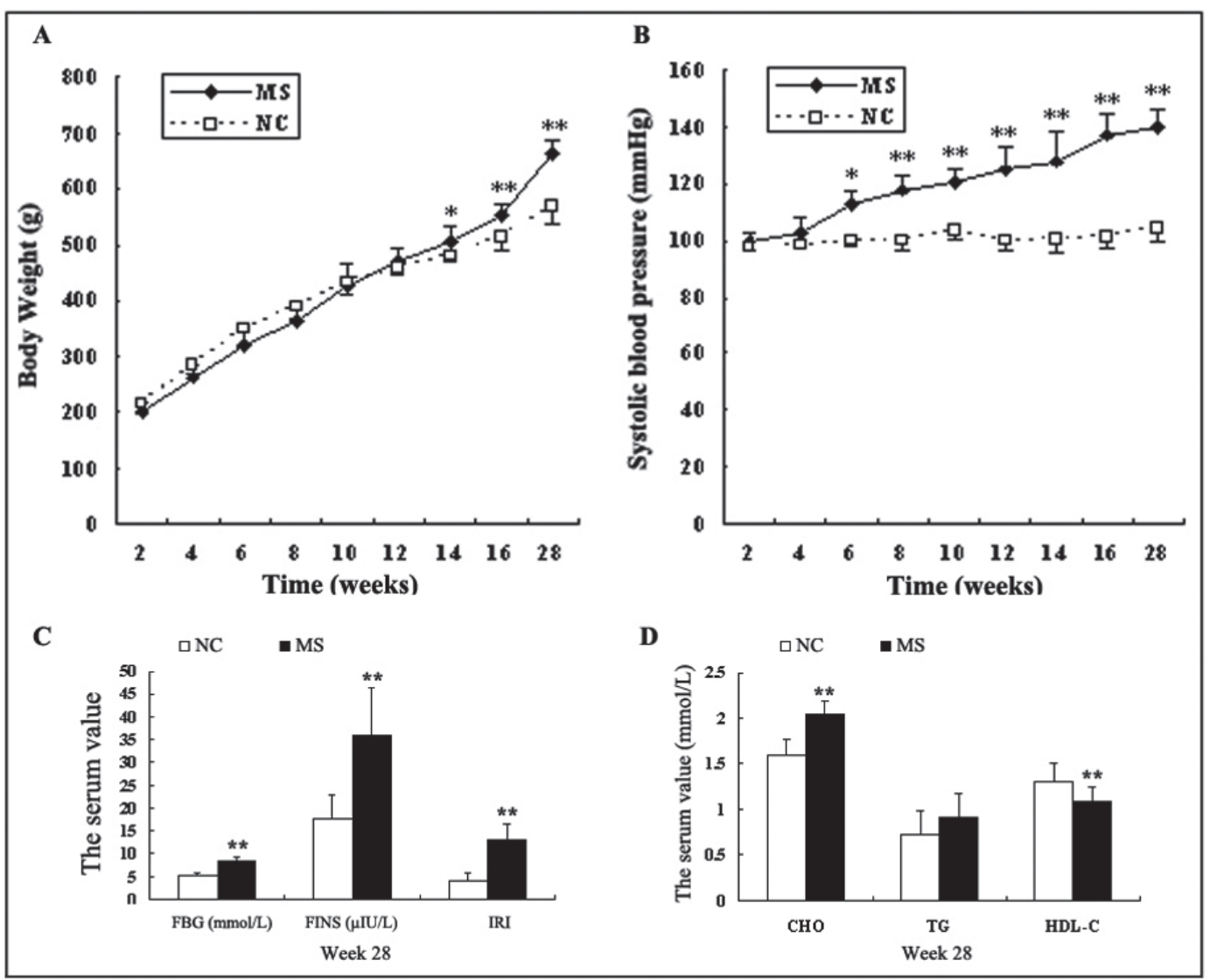

Figure 1. Basic characteristics of MS rats compared with NC rats.

(A) body weight (BW) and (B) systolic blood pressure (SBP) changes from 1 to 28 week. (C) fasting blood glucose (FBG), insulin (FINS) and insulin resistance index (IRI) and (D) cholesterol (CHO), triglycerides (TG) and HDL-C levels at 28 week. Data are shown as means \pm S.D. ${ }^{* * P}<0.01,{ }^{*} P<0.05$ significantly different from the NC group.

Student's $t$ test for two groups, or one-way ANOVA with Newman-Keuls multiple comparison test for more than two groups. A probability value of $P<0.05$ was considered statistically significant. The above statistical analyses were performed using SPSS 13.0.

\section{RESULTS}

\section{Characteristics of MS rats}

MS rats were found to develop insulin resistance, hypercholesterolemia, low HDL-C, hyperglycemia, hypertension and obesity compared to the NC group (Fig. 1).

\section{Echocardiographic features of MS rat heart}

As shown in Table 1, the intraventricular septal wall (IVSW) and left ventricular posterior wall thickness (LVPW) increased in the left ventricle (LV) geometry of MS rats ( $v$ s control rats, $P<0.01$ ), demonstrating eccentric LV hypertrophy. In comparison, the left ventricular enddiastolic dimension (LVEDd), left ventricular end-systolic dimension (LVESd) and fractional shortening (FS, \%) were not significantly changed in the MS rats $(P>0.05)$. Moreover, the $E / A$ ratio and the $E$-wave deceleration time (DT) was significantly higher in MS group compared with the NC group, suggesting reduced diastolic function in the MS rat heart. There was also a significnat difference of the integrated backscatter percent (IBS\%) and cyclic variation of IBS (CVIB) in LVPW and IVS between the NC and MS groups $(P<0.01)$, demonstrating changes of collagen contents in MS rat heart.

\section{Changes of myocardial ultrastructure in MS rats}

TEM showed disruption of myofibers and swollen mitochondria in the MS rat heart (Fig. 2).

\section{Collagen contents measured by Sirius Red and Masson staining}

As shown in Fig. 3, Sirius Red and Masson staining revealed an increase of interstitial fibrosis in the myocardium of MS rats compared with the NC group.

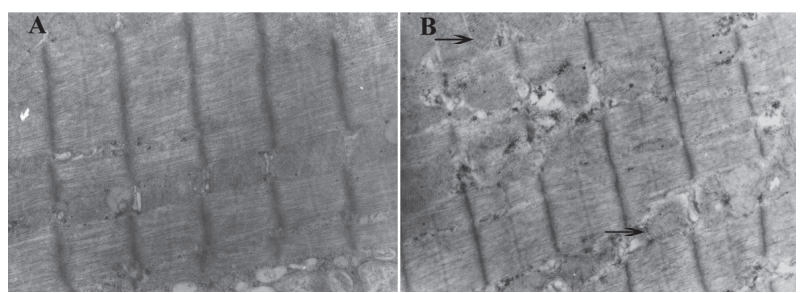

Figure 2. Electron microscopy of myocardium in rats.

(A) NC group. The sarcomere arranged regularly, and the mitochondria of normal size and abundance. (B) MS group. Note the interrupted myofibers and swollen mitochondria shown by arrows. Original magnification: $10000 \times$. 
Table 1. Echocardiographic findings in NC and MS rats at week 1 and 28

\begin{tabular}{|c|c|c|c|c|}
\hline & \multicolumn{2}{|l|}{ Week 1} & \multicolumn{2}{|l|}{ Week 28} \\
\hline & NC & MS & $\mathrm{NC}$ & MS \\
\hline LVEDd (mm) & $4.52 \pm 0.22$ & $4.64 \pm 0.23$ & $5.25 \pm 0.42$ & $5.40 \pm 0.40^{*}$ \\
\hline LVESd (mm) & $1.94 \pm 0.10$ & $1.91 \pm 0.10$ & $2.22 \pm 0.18$ & $2.31 \pm 0.22^{*}$ \\
\hline LVPW (mm) & $1.33 \pm 0.05$ & $1.33 \pm 0.08$ & $1.80 \pm 0.10$ & $2.16 \pm 0.09^{* \# \#}$ \\
\hline IVSW (mm) & $1.27 \pm 0.05$ & $1.29 \pm 0.06$ & $1.78 \pm 0.08$ & $2.14 \pm 0.11^{* \# \#}$ \\
\hline FS & $0.57 \pm 0.02$ & $0.59 \pm 0.02$ & $0.58 \pm 0.03$ & $0.57 \pm 0.04$ \\
\hline$E / A$ & $2.07 \pm 0.24$ & $2.16 \pm 0.31$ & $2.03 \pm 0.20$ & $1.35 \pm 0.22^{* \# \#}$ \\
\hline DT (ms) & $34.70 \pm 4.09$ & $35.33 \pm 5.03$ & $37.07 \pm 4.77$ & $43.47 \pm 4.02^{* \#}$ \\
\hline IBS $\%_{\text {LVPW }}$ & $0.31 \pm 0.05$ & $0.35 \pm 0.08$ & $0.34 \pm 0.08$ & $0.63 \pm 0.11^{* \# \#}$ \\
\hline CVIB $_{\text {LVPW }}$ & $8.40 \pm 1.04$ & $8.39 \pm 1.49$ & $8.09 \pm 1.27$ & $4.45 \pm 1.23^{* \# \#}$ \\
\hline IBS\% ${ }_{\text {IVS }}$ & $0.27 \pm 0.05$ & $0.30 \pm 0.05$ & $0.32 \pm 0.06$ & $0.54 \pm 0.10^{* \# \#}$ \\
\hline $\mathrm{CVIB}_{\text {IVS }}$ & $7.82 \pm 1.10$ & $7.47 \pm 1.48$ & $7.51 \pm 1.50$ & $4.21 \pm 1.08^{* \# \#}$ \\
\hline
\end{tabular}

Results are shown as mean \pm S.D. LVEDd, left ventricular end-diastolic dimension; LVESd, left ventricular end-systolic dimension; LVPW, left ventricular posterior wall thickness; IVSW, intraventricular septal wall; FS, fractional shortening; E/A, ratio of peak early diastolic filling velocity to peak velocity at atrial contrations; DT, E-wave deceleration time; IBS\%, the integrated backscatter percent in LVPW and IVS; CVIB, cyclic variation of IBS. ${ }^{*} P<0.01$ compared to 1 st week in the MS group. $\# P<0.05$, $\# P<0.01$ compared to NC group. Each group comprised ten rats.

\section{IHC analysis of myocardial phospho-AKT and total AKT}

Phospho-AKT was present only sporadically in the myocardium of the MS group, but phospho-AKT staining was predominatly located in the endochylema of cardiocytes in the NC group (Fig. 4B). Total AKT was present similarly in the myocardium of two groups (Fig. 4A).

\section{Localization of apoptosis by TUNEL assay}

Figure 4C shows TUNEL positive cells. Compared with the NC group, the percentage of positive cells relative to normal cardiocytes was significantly increased in the MS group $(P<0.01)$ (Fig. 4D).

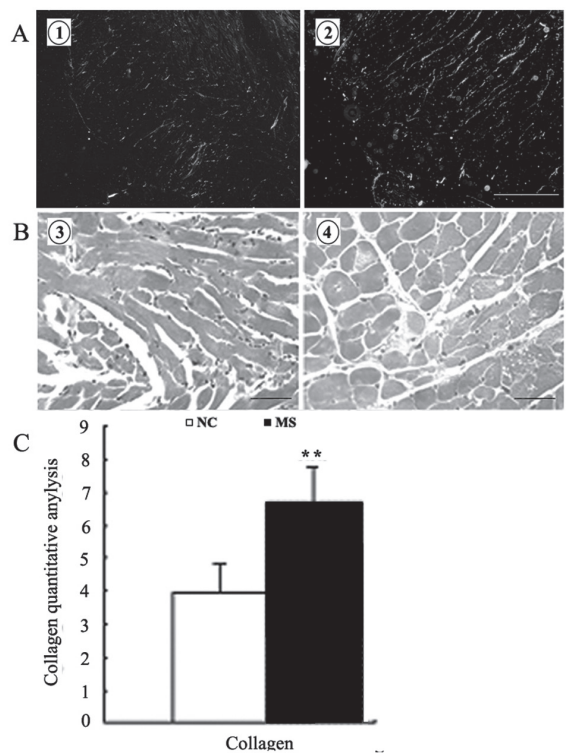

Figure 3. Collagen contents measured by Sirius Red and Masson staining.

(A) Representative Sirius Red staining photomicrographs. 1, NC group. 2, MS group. Original magnification, $400 \times$, bar $=100 \mu \mathrm{m}$. (B) Representative Masson staining photomicrographs. 3, NC group. 4, MS group. Original magnification: $400 \times$, bar $=50 \mu \mathrm{m}$. (C) Bar graphs summarizing the collagen data. Data are shown as means \pm S.D. ${ }^{* *} P<0.01$ significantly different from the NC group.

\section{Western blot analysis of MYPT-1, IRS-1 and AKT}

The expression levels of phosphorylated MYPT-1 (Thr853, Thr696), AKT (Ser473) and IRS-1 (Ser307) are shown in Fig. 5. In the MS group, phosphorylated MYPT-1/ MYPT-1 and phosphorylated IRS-1/ IRS1 levels were significantly higher than those in the NC group, and phosphorylated AKT /AKT levels significantly lower $(P<0.01)$.

\section{DISCUSSION}

MS includes many metabolic disorders, such as obesity, low HDL-C, high triglycerides and impaired glucose tolerance (Norenberg \& Rao, 2007; Tsujimoto \& Shimizu, 2007). In the current study, we created a rat model of MS with the following features: 1) significantly increased body weight, 2) increased systolic blood pressure, 3) increased cholesterol and decreased HDL-C, and 4) increased glucose and insulin levels. Because those trends resembled those reported in human MS patients, those rats thus represent a good model of human MS.

Myocardial impairment in MS patients ultimately results from abnormal cellular metabolism in response to insulin resistance. As myocytes rarely proliferate in adult cardiac muscles, the loss of cardiocytes and the irreversible changes of their morphology would eventually compromise overall heart function. In our study, TUNEL staining demonstrated considerable apoptosis of cardiocytes, and TEM analysis showed pronounced interruption of myofibers and swollen mitochondria, all of which have been implicated in the development of cardiac dysfunction. Mitochondria are vital for cell life, as they represent the principal source of cellular energy (Norenberg \& Rao, 2007; Vial G et al., 2010). Recent studies have demonstrated that improving mitochondrial permeability and swelling could inhibit apoptosis and mediate cardioprotection (Hadzimichalis et al., 2007; Tsujimoto \& Shimizu, 2007). Thus, mitochondrial swelling can be regarded as a characteristic of irreversibly injured myocytes (Jennings \& Reimer, 1991). Furthermore, disrupted myofibers directly affect cardiac diastolic and systolic dysfunction, manifested by decreased compliance, prolonged 


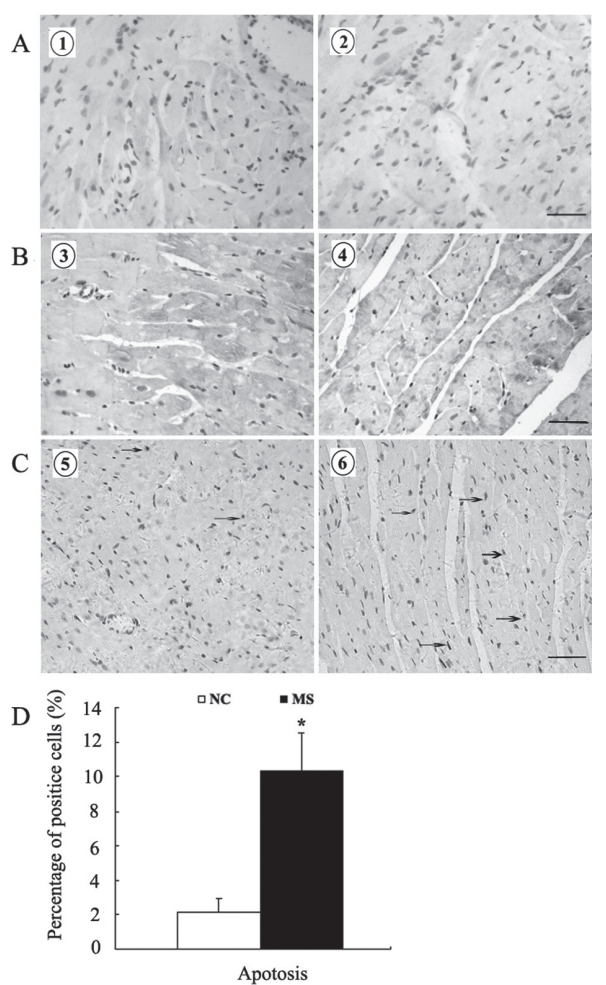

Figure 4. Representative photomicrographs of total AKT and phospho-Akt expression in the myocardium by immunostaining and localization of apoptosis by TUNEL assay.

(A) Representative IHC photomicrographs of total AKT expression in the myocardium. 1, NC group. 2, MS group. Original magnification: $400 \times$, bar $=50 \mu \mathrm{m}$. (B) Representative IHC photomicrographs of phospho-AKT expression in the myocardium. 3, NC group. 4 MS group. Original magnification: $400 \times$, bar $=50 \mu \mathrm{m}$. (C) Representative photomicrographs of apoptotic bodies (pointed by arrows) in the myocardium. 5, NC group. 6, MS group. Original magnification: $400 \times$, bar $=50 \mu \mathrm{m}$. (D) Bar graphs summarizing the apoptosis data. Data are shown as means \pm S.D. ${ }^{*} P<0.01$ significantly different from the NC group.

myocardial relaxation, and left ventricular ejection inability (Lin et al., 2007). At the same time, we found the eccentric hypertrophy and reduced diastolic function of LV by echocardiogram study. These changes are probably due to the inhibition of the PI3-kinase/AKT pathway, an intracellular signaling pathway previously demonstrated to play significant roles not only in insulin sensitivity, but also in any potential protection against myocardial injury, such as ischaemia/reperfusion injury and diabetes ((Hausenloy \& Yellon, 2004; Mocanu \& Yellon, 2007; Tsang et al., 2005). However, few studies have linked the effects of PI3-kinase/AKT pathway activation to cardioprotection in MS. Here, we have demonstrated apoptosis of cardiocytes, changes in sarcomeric and mitochondrial morphology, cardiac hypertrophy, and reduced diastolic function by inhibition of the PI3-kinase/AKT pathway in MS rats.

MS is an early indicator of diabetes and cardiovascular disease that is associated with major mortality and morbidity (Rasouli et al., 2007). However, the exact pathophysiological events leading to the development of MS remain unknown. ROCK is the immediate downstream target of RhoA, and is involved in diverse cellular functions, including smooth muscle contraction, actin cytoskeletal organization, and gene expression. Recent studies suggest that the RhoA/ROCK pathway plays a significant pathophysiological role in various aspects of cardiovascular disease. Indeed, ROCK was activated

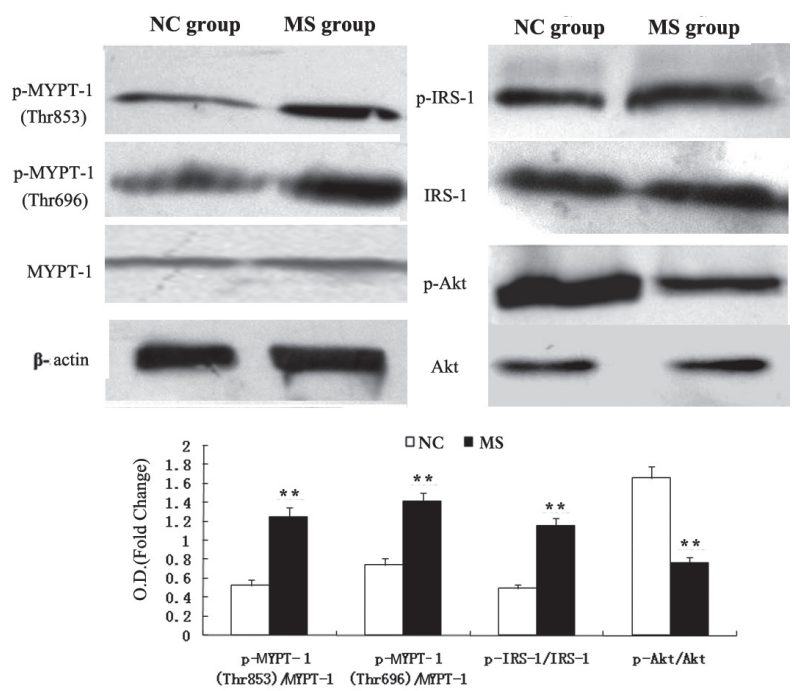

Figure 5. Phospho-MYPT-1(Thr853, Thr696), MYPT-1, phosphoIRS-1 (Ser307), IRS-1, phospho-AKT (Ser473), AKT and $\beta$-actin protein levels in the left ventricle.

Protein levels were determined by Western blotting using special antibodies. $\beta$-actin was a sample loading control. The bar graph displays relative levels of phosphorylated MYPT-1/ MYPT-1, phosphorylated IRS-1/ IRS-1 and phosphorylated AKT /AKT. Data are shown as means \pm S.D. ${ }^{* *} P<0.01$ significantly different from the NC group.

in patients with cardiovascular disease or its associated risk factors, and inhibition of ROCK served as a useful therapeutic approach (Noma et al., 2006; Mustafa S et al., 2010). Although many studies have shown that ROCK activity is increased in the serum of MS patients as well as in smooth muscle cells from MS rats (Liu et al., 2007; Wingard et al., 2007; Lee DH et al., 2009), we demonstrate that ROCK activation was increased in cardiocytes of MS rats and associated with insulin resistance.

Phosphorylated IRS-1 (Ser307) is a key protein involved in insulin resistance, but it has a controversial relationship with ROCK activation. Some studies have shown that ROCK phosphorylates IRS-1, thereby enhancing PI3-kinase activation in adipocytes and muscle cell lines ex vivo (Furukawa et al., 2005). In contrast, phosphorylated IRS-1 led instead to the inhibition of both insulin-induced IRS-1 tyrosine phosphorylation and PI3-kinase/AKT activation in fibroblasts derived from p190B RhoGAP-null mice and in vascular smooth muscle cells ((Begum et al., 2002; Loirand et al.,2006; Sordella et al., 2002). Similarly, our results also indicate that ROCK activity was increased in the hearts of MS rats and that it negatively modulated insulin signaling. Increased ROCK activation was accompanied by IRS-1 serine phosphorylation, which inhibited AKT kinase activation and ultimately resulted in cardiocyte apoptosis and changes to the myocardial structure and function. Thus, insulin signaling in the hearts of MS rats could be attenuated by ROCK activation, implying a negative role for ROCK in insulin resistance.

In summary, this is the first demonstration that ROCK is activated in hearts from rats with MS. Furthermore, ROCK-associated serine phosphorylation of IRS-1 plays a critical role in insulin resistance, suggesting that ROCK may be a promising future therapeutic target in the treatment of MS. At the same time, there were some deficiencies in our study. As only ten MS rats were investigated in this study, we should confirm our findings 
with a larger sample size. Additionally, we did not inhibit ROCK activation with either siRNA technology or specific inhibitors, such as Y27632 or fasudil. We plan to assess the effects of ROCK inhibition in vivo and in vitro in future studies.

\section{Acknowledgements}

We gratefully acknowledge technical assistance from Xiang-Ming Zhang, Fan-Jiang, Xu-Ping Wang, and Hong-Jiang.

This study was supported by the National 973 Basic Research Program of China (2010CB732605), four grants from the Department of Science and Technology of Shandong Province (2011GGH21834, ZR2010HQ059, ZR2010HQ017 and 2008RKB060), three grants from the Health Department of Shandong Province (Shandong Provincial Outstanding Medical Academic Professional Program, 1020 Program, and 2009HD011), two Independent Innovation Foundation of Shandong University (2010TS082 and 2012TS154), and a grant from Jinan Science and Technology Bureau (201102073).

\section{REFERENCES}

Amaravadi R \& Thompson CB (2005) The survival kinases AKT and Pim as potential pharmacological targets. J Clin Invest 115: 26182624.

Asano T, Fujishiro M, Kushiyama A, Nakatsu Y, Yoneda M, Kamata $\mathrm{H} \&$ Sakoda H (2007) Role of phosphatidylinositol 3-kinase activation on insulin action and its alteration in diabetic conditions. Biol Pharm Bull 30: 1610-1616.

Begum N, Sandu OA, Ito M, Lohmann SM \& Smolenski A (2002) Active Rho kinase (ROK-alpha) associates with insulin receptor substrate-1 and inhibits insulin signaling in vascular smooth muscle cells. J Biol Chem 277: 6214-6222.

Bivalacqua TJ, Champion HC, Usta MF, Cellek S, Chitaley K, Webb RC, Lewis RL, Mills TM, Hellstrom WJ \& Kadowitz PJ (2004) RhoA/Rho-kinase suppresses endothelial nitric oxide synthase in the penis: a mechanism for diabetes-associated erectile dysfunction. Proc Natl Acad Sci USA 101: 9121-9126.

Boden G (2006) Fatty acid-induced inflammation and insulin resistance in skeletal muscle and liver. Curr Diab Rep 6: 177-181.

Bonora E (2006) The metabolic syndrome and cardiovascular disease. Ann Med 38: 64-80.

Chitaley K, Weber D \& Webb RC (2001) RhoA/Rho-kinase, vascular changes, and hypertension. Curr Hypertens Rep 3: 139-144.

Fan JG \& Peng YD (2007) Metabolic syndrome and non-alcoholic fatty liver disease: Asian definitions and Asian studies. Hepatobiliary Pancreat Dis Int 6: 572-578.

Ferre P (2007) Insulin signaling and insulin resistance. Therapie 62: $277-284$

Fonseca VA (2003) Management of diabetes mellitus and insulin resistance in patients with cardiovascular disease. Am J Cardiol 92: 50J$60 \mathrm{~J}$.

Furukawa N, Ongusaha P, Jahng WJ, Araki K, Choi CS, Kim HJ, Lee YH, Kaibuchi K, Kahn BB, Masuzaki H, et al. (2005) Role of Rhokinase in regulation of insulin action and glucose homeostasis. Cell Metab 2: 119-129.

Hadzimichalis NM, Baliga SS, Golfetti R, Jaques KM, Firestein BL \& Merrill GF (2007) Acetaminophen-mediated cardioprotection via in- hibition of the mitochondrial permeability transition pore-induced apoptotic pathway. Am J Physiol Heart Circ Physiol 293: H3348-3355.

Hausenloy DJ \& Yellon DM (2004) New directions for protecting the heart against ischaemia-reperfusion injury: targeting the Reperfusion Injury Salvage Kinase (RISK)-pathway. Cardiovasc Res 61: 448-460.

Jennings RB \& Reimer KA (1991) The cell biology of acute myocardial ischemia. Annu Rev Med 42: 225-246.

Lee DH, Shi J, Jeoung NH, Kim MS, Zabolotny JM, Lee SW, White MF, Wei L, Kim YB (2009)Targeted disruption of ROCK1 causes insulin resistance in vivo. J Biol Chem 284: 11776-11780.

Lin G, Craig GP, Zhang L, Yuen VG, Allard M, McNeill JH \& MacLeod KM (2007) Acute inhibition of Rho-kinase improves cardiac contractile function in streptozotocin-diabetic rats. Cardiovasc Res 75: 51-58.

Liu PY, Chen JH, Lin LJ \& Liao JK (2007) Increased Rho kinase activity in a Taiwanese population with metabolic syndrome. $\mathrm{J} A \mathrm{~m}$ Coll Cardiol 49: 1619-1624.

Loirand G, Guerin P \& Pacaud P (2006) Rho kinases in cardiovascular physiology and pathophysiology. Circ Res 98: 322-334.

Mocanu MM \& Yellon DM (2007) PTEN, the Achilles' heel of myocardial ischaemia/reperfusion injury? BrJ Pharmacol 150: 833-838.

Mukai Y, Shimokawa H, Matoba T, Kandabashi T, Satoh S, Hiroki J, Kaibuchi K \& Takeshita A (2001) Involvement of Rho-kinase in hypertensive vascular disease: a novel therapeutic target in hypertension. FASEB J 15: 1062-1064.

Mustafa S, Vasudevan H, Yuen VG, McNeill JH (2010) Renal expression of arachidonic acid metabolizing enzymes and RhoA/Rho kinases in fructose insulin resistant hypertensive rats. Mol Cell Biochem 333: 203-209.

Naik JS, Xiang L \& Hester RL (2006) Enhanced role for RhoA-associated kinase in adrenergic-mediated vasoconstriction in gracilis arteries from obese Zucker rats. Am J Physiol Regul Integr Comp Physiol 290: R154-R161.

Noma K, Oyama N \& Liao JK (2006) Physiological role of ROCKs in the cardiovascular system. Am I Physiol Cell Physiol 290: C661-C668.

Norenberg MD \& Rao KV (2007) The mitochondrial permeability transition in neurologic disease. Neurochem Int 50: 983-997.

Rasouli N, Molavi B, Elbein SC \& Kern PA (2007) Ectopic fat accumulation and metabolic syndrome. Diabetes Obes Metab 9: 1-10.

Sesti G (2006) Pathophysiology of insulin resistance. Best Pract Res Clin Endocrinol Metab 20: 665-679.

Shi J \& Wei L (2007) Rho kinase in the regulation of cell death and survival. Arch Immunol Ther Exp (Warsq) 55: 61-75.

Sordella R, Classon M, Hu KQ, Matheson SF, Brouns MR, Fine B, Zhang L, Takami H, Yamada Y \& Settleman J (2002) Modulation of CREB activity by the Rho GTPase regulates cell and organism size during mouse embryonic development. Dev Cell 2: 553-565.

Tsang A, Hausenloy DJ, Mocanu MM, Carr RD \& Yellon DM (2005) Preconditioning the diabetic heart: the importance of AKT phosphorylation. Diabetes 54: 2360-2364.

Tsujimoto Y \& Shimizu S (2007) Role of the mitochondrial membrane permeability transition in cell death. Apoptosis 12: 835-840.

Uehata M, Ishizaki T, Satoh H, Ono T, Kawahara T, Morishita T, Tamakawa H, Yamagami K, Inui J, Maekawa M, et al. (1997) Calcium sensitization of smooth muscle mediated by a Rho-associated protein kinase in hypertension. Nature 389: 990-994.

Vial G, Dubouchaud H, Leverve XM (2010) Liver mitochondria and insulin resistance. Acta Biochim Pol 57: 389-392.

Wingard C, Fulton D \& Husain S (2007) Altered penile vascular reactivity and erection in the Zucker obese-diabetic rat. J Sex Med 4: $348-362$.

Xie Z, Su W, Guo Z, Pang H, Post SR \& Gong MC (2006) Up-regulation of CPI-17 phosphorylation in diabetic vasculature and high glucose cultured vascular smooth muscle cells. Cardiovasc Res 69: 491-501. 